\title{
Evaluation on Risks in Inter-firm Knowledge Transfer Integrating ANP and BP Neural Network
}

\author{
Chen Guo ${ }^{\mathrm{a}}$, Qi Ershib \\ College of Management and Economics, Tianjin University, Tianjin 300072, China \\ aamigofishchen@163.com, bscholarqi@126.com
}

Keywords: risk evaluation, knowledge transfer, Analytic Network Process, BP neural network

\begin{abstract}
The risk attitude of the knowledge sender influences risks in inter-firm knowledge transfer. By introducing the risk attitude of the knowledge sender, a new risk evaluation method integrating Analytic Network Process and BP neural network was proposed. An example was applied to demonstrate this method by using SuperDecisions and MATLAB. The results showed that it is necessary to consider the risk attitude of the knowledge sender when evaluating risks in inter-firm knowledge transfer and this new method can evaluate risks well.
\end{abstract}

\section{Introduction}

In the era of knowledge economy, the enterprise not only has to update internal knowledge, but also has to absorb external knowledge to enhance its competitiveness. Inter-firm knowledge transfer becomes more and more popular. However, the knowledge sender's knowledge may be lost or stolen in inter-firm knowlede transfer ${ }^{[1]}$. This problem has attracted the attention of many scholars ${ }^{[2,3]}$. They generally believed that an effective evaluation on risks in inter-firm knowledge transfer is extremely meaningful. Some scholars began to study this problem. Yan Wei et al. used fuzzy comprehensive evaluation method to evaluate intellectual property risks in dynamic alliance ${ }^{[4]}$. Xiao Lingnuo et al. used BP neural network to assess risks in industry-university-research knowledge innovation alliance $^{[5]}$. Li Bozhou et al. evaluated risks in knowledge transfer between cooperation innovation enterprises $^{[6]}$. However, these studies did not reflect the influence of the knowledge sender's risk attitude to risks in inter-firm knowledge transfer.

An enterprise's risk attitude is its attitude to uncertain situation ${ }^{[7,8]}$. Some scholars found that the enterprise's risk attitude plays an important role when the enterprise faces risks. Bao Xing et al. verified the manager's risk attitude can influence its emergency management by affecting its investment on rapid support ability and system capacity ${ }^{[9,10]}$. Du Jianguo et al. found the risk attitudes of the supply chain members can affect the risk of supply chain disruption ${ }^{[11]}$. However, the importance of the knowledge sender's risk attitude to risks in inter-firm knowledge transfer has not been noticed. In order to make up for this shortage, we took the risk attitude of the knowledge sender as a risk evaluation index. By considering the knowledge sender's risk attitude's influence on other risk evaluation indexes and risks in inter-firm knowledge transfer, we developed a new risk evaluation system.

Among all methods, Analytic Network Process (ANP) combines qualitative analysis with quantitative analysis and can reflect the dependent relationship among all elements ${ }^{[12]}$. BP neural network is designed to solve mapping approaching problem whose rule implied in numerous disorganized data. Because risks in inter-firm knowledge transfer are affected by a lot of elements implied in large amounts of disorganized information in a complex way. This paper introduces the risk attitude of the knowledge sender and develops a method of evaluating risks in inter-firm knowledge transfer integrating ANP and BP neural network. Section 2 briefly describes the concepts of ANP and BP neural network. Section 3 presents the process of evaluating risks in inter-firm knowledge transfer integrating ANP and BP neural network. An example is applied to demonstrate this method by using SuperDecisions and MATLAB in Section 4. Section 5 gives the conclusions of this paper. 


\section{ANP and BP neural network}

The network structure of ANP is shown in Fig. 1 (Here is given the evaluation on risks in inter-firm knowledge transfer as an example. And the network structure was built by using SuperDecisions, which is a software developed by Saaty according to the theory of ANP). It includes a control layer and a network layer. In this paper, the control layer only contains a goal and the network layer contains three element groups. The goal is influenced by all element groups and element groups may influence each other. By successively building judgment matrices, super matrices, weighted super matrices, and limit super matrices, we can finally obtain the weights of the elements in the network layer.

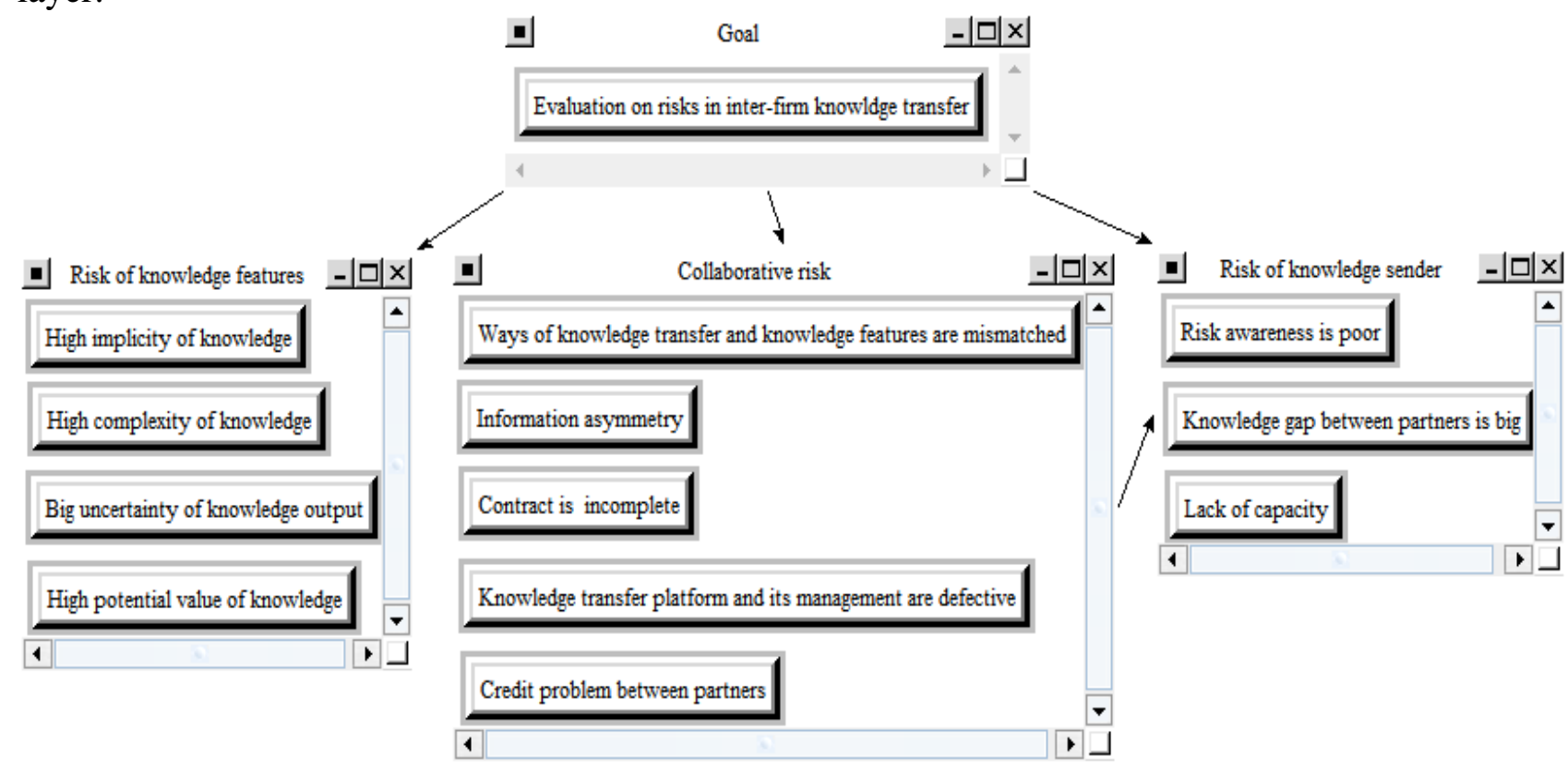

Fig. 1 Network structure of evaluation on risks in inter-firm knowledge transfer

Fig. 2 shows the topological structure of BP neural network ${ }^{[13]}$. It contains input layer, hidden layer, and output layer (Here is given the evaluation on risks in inter-firm knowledge transfer as an example). Its theory is to find out the nonlinear relationship among data by learning, which includes information dissemination and error back propagation ${ }^{[14]}$.

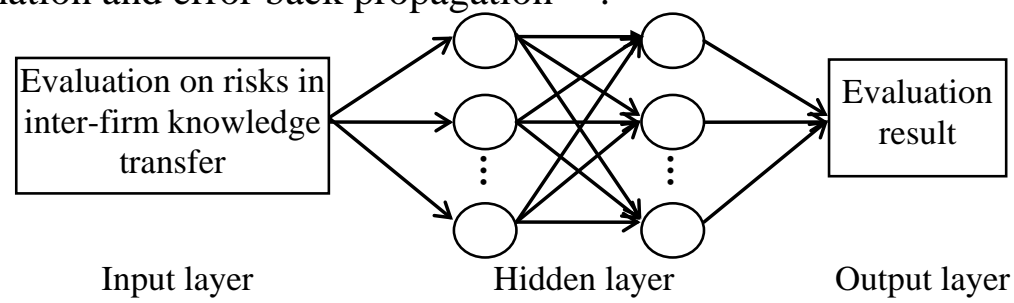

Fig. 2 Evaluation model of risks in inter-firm knowledge transfer based on BP neural network.

This paper provides a new approach to evaluating risks in inter-firm knowledge transfer integrating ANP and BP neural network, which can weaken the strong subjectivity of ANP and the strong objectivity of BP neural network. This method is mainly developed by BP neural network and assisted by ANP. And its evaluation results are weighted sums of the evaluation results of BP neural network and those of ANP.

\section{Evaluating risks using ANP and BP neural network}

The specific process of evaluating risks in inter-firm knowledge transfer integrating ANP and BP neural network is described below.

Evaluating risks using ANP. Firstly, we built the original risk evaluation index system through Delphi method. Then, we established the network structure of this problem. By paired comparing the importances of the indexes in the same group to the index in another group, we built judgment 
matrices. By calculating the maximum eigenvalues and corresponding eigenvectors of the judgment matrices whose inconsistencies are acceptable, we got super matrices. By weighting super matrices, we got weighted super matrices which can reflect the dependent relationship among all indexes. And by calculating limit super matrices, we can finally obtain the weights of the indexes in the network layer. The indexes with larger weights are key evaluation indexes. Finally, by calculating the sum of each key evaluation index's score multiplied by its weight, we got the evaluation result of each sample.

Evaluating risks using BP neural network. Nielson demonstrated a three-layer BP neural network can complete any mapping from $\mathrm{n}$ dimension to $\mathrm{m}$ dimension ${ }^{[15]}$. Thus we developed a three-layer BP neural network. The number of nodes in input layer is the number of key evaluation indexes. According to the computer simulation, we determined the number of nodes in hidden layer is 8. We determined the number of nodes in output layer is 1 for the evaluation result of each sample is unique. And each neuron's output function is a sigmoid function:

$$
\begin{aligned}
& I=\sum_{j=0}^{n} w_{j} x_{j} \\
& \mathrm{y}=\mathrm{f}(\mathrm{I})=\frac{1}{1+\exp (-\mathrm{I})}
\end{aligned}
$$

$x_{j}$ is input signal, $w_{j}$ is the strength of joint among neurons, $f(I)$ is the output function.

Finally we divided the samples data into training set and testing set, and evaluated the risks of samples in testing set using the BP neural network trained by training set.

Evaluating risks using ANP and BP neural network. Using the weights discussed by experts to calculate weighted sums of the evaluation results of ANP and those of BP neural network, we can get the final evaluation results.

\section{Example}

We studied 25 cases of inter-firm knowledge transfer in Tianjin and took these 25 cases as samples to evaluate their risks. 12 important evaluation indexes were selected through Delphi method. The network structure of this problem is shown in Fig. 1. Judgment matrices were established then. By using SuperDecisions, we can get limit super matrices and calculate weights of all indexes (as shown in Table 1). 10 key evaluation indexes can be obtained by eliminating 2 indexes with smaller weights.

\begin{tabular}{|c|c|c|c|}
\hline One class index & Two class index & $\begin{array}{l}\text { Local } \\
\text { weight }\end{array}$ & $\begin{array}{c}\text { Final } \\
\text { weight }\end{array}$ \\
\hline \multirow{4}{*}{$\begin{array}{l}\text { Risk of } \\
\text { knowledge } \\
\text { features }\end{array}$} & Hgih implicity of knowledge & 0.37501 & 0.023438 \\
\hline & High complexity of knowledge & 0.37501 & 0.023438 \\
\hline & Big uncertainty of knowledge output & 0.12499 & 0.007812 \\
\hline & High potential value of knowledge & 0.12499 & 0.007812 \\
\hline \multirow{5}{*}{$\begin{array}{c}\text { Collaborative } \\
\text { risk }\end{array}$} & Ways of knowledge transfer and knowledge features are mismatched & 0.0827 & 0.025845 \\
\hline & Information asymmetry & 0.15888 & 0.049651 \\
\hline & Contract is incomplete & 0.29544 & 0.092325 \\
\hline & Knowledge transfer platform and its management are defective & 0.04637 & 0.01449 \\
\hline & Credit problem between partners & 0.4166 & 0.130189 \\
\hline \multirow{3}{*}{$\begin{array}{l}\text { Risk of } \\
\text { knowledge } \\
\text { sender }\end{array}$} & Risk awareness is poor & 0.37824 & 0.236399 \\
\hline & Knowledge gap between partners is big & 0.05236 & 0.032728 \\
\hline & Lack of capacity & 0.5694 & 0.355873 \\
\hline
\end{tabular}

Table 1 Weights of evaluation indexes of risks in inter-firm knowledge transfer based on ANP

We divided key indexes into 5 grades: very unfit, unfit, moderate, fit, and very fit. Scores of 5 grades in turn are $0,0.25,0.5,0.75$, and 1.5 samples randomly selected from 25 samples are testing set. The sum of each key index's score multiplied by its weight given by ANP is the evaluation result of each sample in testing set based on ANP. 
The other 20 samples are training set. Take the evaluation results and scores of key indexes judged by experts of samples in training set as learning data to train the BP neural network by using MATLAB. The main program code is:

$$
\text { net = newff([0 1;0 1;0 1;0 1;0 1;0 1;0 1;0 1;0 1; } 0 \text { 1],[10 } 8 \text { 1],\{'tansig' 'purelin'\}); }
$$

net.trainParam.epochs $=50$

Evaluation results of samples in testing set can be obtained by entering these samples data into the trained BP neural network. We divided risk evaluation result into 5 levels: very low, low, moderate, high, and very high. Scores ranges of 5 levels in turn are 0-0.2, 0.2-0.4, 0.4-0.6, 0.6-0.8, and 0.8-1.The evaluation results and scores of key indexes of 5 samples in testing set are shown in Table 2.

Table 2 Evaluation results of 5 samples based on BP neural network

\begin{tabular}{cccccc}
\hline Input index & Sample 1 & Sample 2 & Sample 3 & Sample 4 & Sample 5 \\
\hline Lack of capacity & 0.5 & 0.75 & 0.5 & 0.25 & 0.5 \\
Risk awareness is poor & 0.5 & 0.75 & 0.75 & 0.5 & 0.25 \\
Credit problem between partners & 0.25 & 1 & 0.25 & 0.5 & 0.25 \\
Contract is incomplete & 0.25 & 1 & 0.5 & 0.25 & 0.5 \\
Information asymmetry & 0.5 & 0.5 & 0.75 & 0.75 & 0.5 \\
Knowledge gap between partners is big & 0.25 & 1 & 0.5 & 0.75 & 0.25 \\
Ways of knowledge transfer and & 0.25 & 0.5 & 1 & 0.75 & 0.25 \\
knowledge features are mismatched & & & & 0.75 & 0.5 \\
High implicity of knowledge & 0.5 & 0.75 & 0.75 & 0.5 & 0 \\
High complexity of knowledge & 0 & 0 & 0.5 & 0.5 & 0.5 \\
Knowledge transfer platform and its & 0.75 & 0.75 & 0.5 & 0.25 & 0.5 \\
management are defective & 0.45 & 0.72 & 0.51 & 0.47 & 0.35 \\
Target outputs & 0.47 & 0.69 & 0.44 & 0.50 & 0.34 \\
Actual outputs & Moderate & High & Moderate & Moderate & Low \\
Level & 1 &
\end{tabular}

Among 5 samples in Table 2, there is only 1 sample whose actual output is far different from the target output. Thus the method can evaluate risks well.

As experts suggested evaluation results would be more scientific if they are sums of the evaluation results based on ANP multiplied by $45 \%$ and those based on BP neural network multiplied by 55\%. New evaluation results of 5 samples are shown in Table 3.

Table 3 Evaluation results of 5 samples based on different methods

\begin{tabular}{cccccc}
\hline Method & Sample 1 & Sample 2 & Sample 3 & Sample 4 & Sample 5 \\
\hline ANP & 0.42 & 0.78 & 0.56 & 0.41 & 0.38 \\
BP neural network & 0.47 & 0.69 & 0.44 & 0.50 & 0.34 \\
ANP-BP neural network & 0.448 & 0.731 & 0.494 & 0.460 & 0.358 \\
Target outputs & 0.45 & 0.72 & 0.51 & 0.47 & 0.35 \\
Level & Moderate & High & Moderate & Moderate & Low \\
\hline
\end{tabular}

By comparing evaluation results of 5 samples in Table 3, we can find that:

(1) Evaluation results based on ANP that considering the influence of the risk attitude of the knowledge sender to other evaluation indexes and risks are not very different from the evaluation results based on BP neural network. That means the risk attitude of the knowledge sender has great influence on other evaluation indexes and risks. Thus it is necessary to consider the risk attitude of the knowledge sender when evaluating risks in inter-firm knowledge transfer.

(2) Evaluation results based on ANP-BP neural network are the results closest to the target outputs. The method integrating ANP and BP neural network can evaluate risks well.

\section{Conclusion}

This paper takes the risk attitude of the knowledge sender as an evaluation index and skillfully combines ANP with BP neural network to evaluate risks in inter-firm knowledge transfer. This 
method not only uses the advantages of ANP, especially it can reflect the dependent relationship among all elements; but also uses the objectivity of BP neural network. And it weakens both the strong subjectivity of ANP and the strong objectivity of BP neural network. Practice shows that, it is necessary to consider the risk attitude of the knowledge sender when evaluating risks in inter-firm knowledge transfer and the new evaluation method developed in this paper can evaluate risks well. This paper still has some deficiencies: the weights used to calculate the final evaluation results based on ANP-BP neural network may be subjective, a more objective method need to be found to optimize these weights. And this paper only solve the problem of evaluating risks in inter-firm knowledge transfer, future research may consider how to evaluate risks in inter-firm knowledge sharing.

\section{References}

[1] P.M. Norman, Protecting knowledge in strategic alliances: resource and relational characteristics, J. Journal of High Technology Management Research. 13 (2002) 177-202.

[2] J. Hagedoorn, Understanding the rationale of strategic technology partnering: interorganizational modes of cooperation and sectoral differences, J.Strategic Management Journal. 14 (1993) 371-385.

[3] P. Quintas, P. Lefrere, G. Jones, Knowledge management: a strategic agenda, J. Long Range Planning. 30 (1997) 385-391.

[4] Yan Wei, Chen Yan, Chen Linbo, Theoretical analysis and case study on evaluation of intellectual property risks in dynamic alliance, J. Science \& Technology Progress and Policy. 26 (2009) 120-123.

[5] Xiao Lingnuo, Shi Jianfeng, Sun Yuzhong, Risk assessment of industry-university-research knowledge innovation alliance based on BP neural network algorithm, J. China Soft Science. 12 (2001) 173-179.

[6] Li Bozhou, Xu Guangyu, Risk evaluation on inter-firm knowledge transfer in collaborative innovation based on method set, J. Science \& Technology Progress and Policy. 31 (2014) 112-117.

[7] E.U. Weber, R.A. Miiliman, Perceived risk attitudes: relating risk perception to risky choice, J. Management Science. 43 (1997) 123-144.

[8] W.M. Goldstein, H.J. Einhorn, Expression theory and the preference reversal phenomenal, J. Psychological Review. 94 (1987) 236-254.

[9] Bao Xing, Sun Qi, The influence of manager's risk attitude to procurement decision when system's capacity was partially injured, J. Chinese Journal of Management Science. 20 (2012) 87-92.

[10] Bao Xing, Ji Jianhua, Disrupting capacity management of service operation system under decision-maker’s risk attitude, J. Journal of Systems \& Management. 18 (2009) 555-561.

[11] Du Jianguo, Wan Yahong, Hou Yunzhang, Study on behavior evolution based on the risk attitude of the supply chain members, J. Journal of Systems \& Management. 22 (2013) 828-834, 840.

[12] T.L. Saaty, The analytic network process: decision making with dependence and feedback, RWS Publications, Pittsburgh, 2001.

[13] Wang Keliang, Yang Li, Zha Fugeng, Desigh of the post evaluation system based on BP neural network, J. Enterprise Economics. 12 (2007) 30-33.

[14] Ge Zhexue, Sun Zhiqiang, Neural network principle and its realization by MATLABR2007, Electronic Industry Press, Beijing, 2008.

[15] R.Hecht Nielsen, Neurocomputing. Addison Wesley, 1990. 